\title{
Heavy photons at coupled-cavity waveguide band edges in a three-dimensional photonic crystal
}

\author{
Mehmet Bayindir* and E. Ozbay \\ Department of Physics, Bilkent University, Bilkent, 06533 Ankara, Turkey
}

(Received 10 April 2000)

\begin{abstract}
We report on measurements of delay time corresponding to evanescent coupled-cavity modes in threedimensional photonic crystals. By creating a defect inside the crystal, photons are confined within a boxlike cavity of volume $\sim(\lambda / 2)^{3}$. It is observed that photon lifetime increases drastically and group velocity of photons tends towards zero at the waveguiding band edges of the periodic coupled cavities. Experimental results are well explained within the classical wave analog of the tight-binding picture. Observation of the extremely small group velocity and long photon lifetime at the coupled-cavity waveguide band edges have important potential applications.
\end{abstract}

In the last several years, there has been much interest in physics and applications of spatially periodic dielectric or metallic structures, photonic band gap materials, which inhibit propagation of the electromagnetic (EM) waves within a certain range of wavelengths in all directions. ${ }^{1,2}$ The intense experimental and theoretical investigations continue on localization of light, ${ }^{3,4}$ tunneling phenomena in photonic systems, ${ }^{5-8}$ alteration (i.e., inhibition and enhancement) of spontaneous emissions. ${ }^{9-11}$

By introducing a defect into a photonic crystal, photons with certain wavelengths can locally be trapped inside the defect volume, which is analogous to the localized impurity states in a semiconductor. ${ }^{12}$ Recently, we experimentally and theoretically investigated splitting of the EM modes due to coupling between the evanescent cavity modes, ${ }^{13}$ and guiding and bending of EM waves through periodic coupled cavities in three-dimensional (3D) photonic crystals. ${ }^{14}$ In this paper, we measure the delay time corresponding to localized cavity modes inside a 3D photonic crystal. We report on observation of heavy photons at the waveguiding band edges of the coupled-cavity array by measuring photon lifetime and group velocity of photons along the cavity array [Fig. 1(a)]. A formula for the photon lifetime is derived within the tightbinding (TB) formalism ${ }^{13,15-18}$ that well describes the experimental results. For the two coupled-cavity case, it is also observed that photons in the antibonding mode spend more time inside the cavities compared to the bonding mode.

Heavy photons or photons with a extremely low group velocity play a critical role in enhancing the efficiency of nonlinear processes, ${ }^{21,20,17}$ and gain enhancement in the photonic band edge laser. ${ }^{19}$ Moreover, the spontaneous emission rate can be increased since the effective gain is proportional to $1 / v_{g} .{ }^{20}$ The low group velocity was proposed near the band edges of one-dimensional photonic band-gap structures. ${ }^{19,21}$ Recently, Vlasov et al. have observed that the optical pulses significantly slow down at the $3 \mathrm{D}$ photonic crystal band edges in the visible spectrum. ${ }^{22}$

A layer-by-layer dielectric based photonic crystal ${ }^{23,24}$ was used to construct the coupled cavities. The crystal consists of square shaped alumina rods having a refractive index 3.1 at the microwave frequencies and dimensions $0.32 \mathrm{~cm} \times 0.32$ $\mathrm{cm} \times 15.25 \mathrm{~cm}$. A center-to-center separation between the rods of $1.12 \mathrm{~cm}$ was chosen to yield a dielectric filling ratio of $\sim 0.26$. The unit cell consists of four layers having the symmetry of a face-centered tetragonal (fct) crystal structure. The crystal exhibits a three-dimensional photonic band gap extending from 10.6 to $12.8 \mathrm{GHz}^{24,25}$

The experimental setup consists of a HP 8510C network analyzer and microwave horn antennas to measure the

(a)

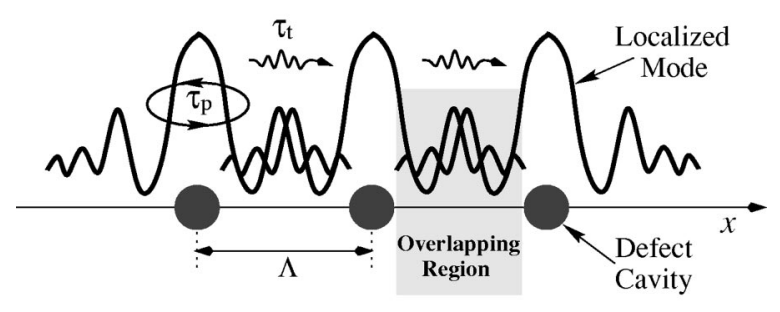

(b)

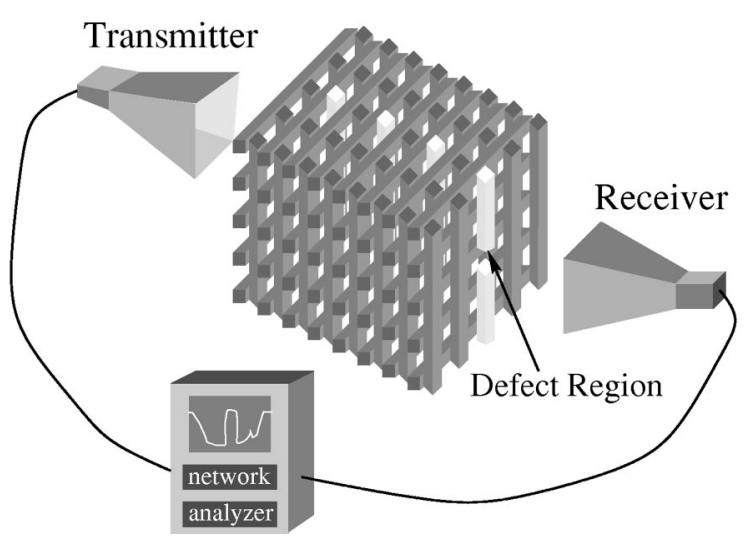

FIG. 1. (a) Schematics of propagation of photons through localized coupled-cavity modes within a photonic crystal. Amplitude of the strongly localized EM modes is enhanced inside each cavity, which leads to high $Q$ factors and long lifetime $\tau_{p}$. The transit time is the sum of the escape rate of photons from the cavity $\left(\tau_{p}\right)$ and the tunneling time between the cavities $\left(\tau_{t}\right)$. (b) Experimental setup for measuring the transmission-amplitude and transmission-phase spectra of the coupled cavities in 3D photonic crystals. By removing some portion of a single rod, we can confine photons within a volume $\sim(\lambda / 2)^{3}$. 


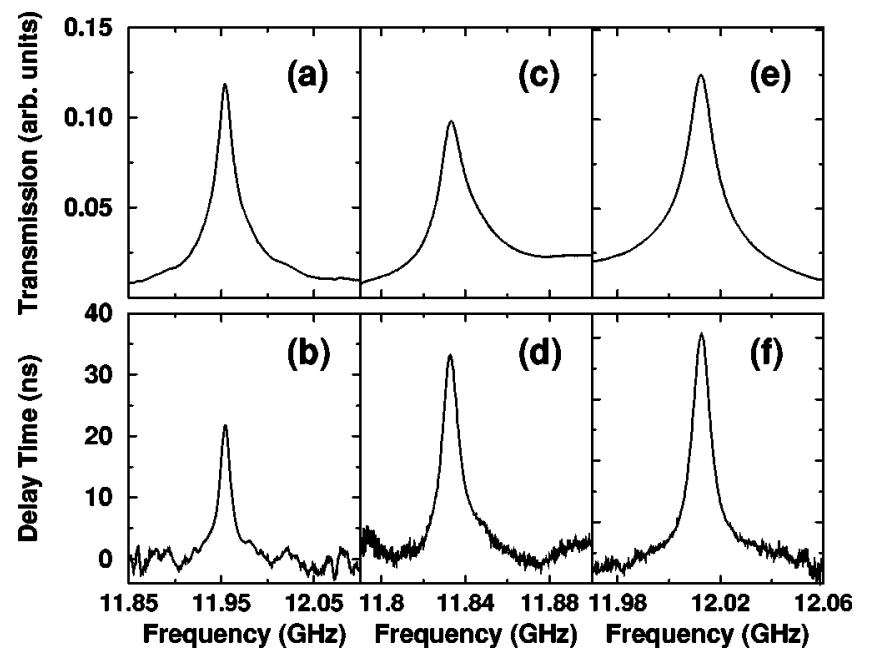

FIG. 2. (a) Transmission through a photonic crystal with a single defect. Transmission has a peak at $\Omega=11.954 \mathrm{GHz}$ with $Q$ $=940$. (b) Photon lifetime, $\tau_{p}=\partial \varphi / \partial \omega$, as a function of frequency. Corresponding lifetime at the resonance frequency is $23 \mathrm{~ns}$. Transmission spectrum (c) and (e) and delay time (d) and (f) for two coupled cavities with five unit cell crystal. The bonding and antibonding resonances occurs at $\omega_{\mathrm{B}}=11.833 \mathrm{GHz}$ and $\omega_{\mathrm{A}}=12.012$ $\mathrm{GHz}$, with $\tau_{p}^{\mathrm{B}}=34 \mathrm{~ns}$ and $\tau_{p}^{\mathrm{A}}=37 \mathrm{~ns}$. The delay time corresponding to the antibonding mode is always longer than the delay time of the bonding mode.

transmission-amplitude and transmission-phase spectra [Fig. 1(b)]. Removing some portion of a single rod from an otherwise perfect crystal leads to confined modes with high quality-factors, $Q=\Delta \lambda / \lambda_{0}$, around $1000 .{ }^{26}$ Photons of wavelength $\lambda \sim 2.5 \mathrm{~cm}$ are confined within a very small volume order of $V_{L} \sim 1 \mathrm{~cm}^{3}$. The electric-field polarization vector of the incident EM field was always parallel to the rods of the defect lines for all measurements.

We first measured the transmission spectra and the delay time for a four unit cell crystal with a single cavity. Delay time or photon lifetime is defined as $\tau_{p}=\partial \varphi / \partial \omega$ and that can be obtained from the transmission-phase measurements. Here $\varphi$ is the net phase difference between the phase of the EM wave propagating through the photonic crystal and the phase of the EM wave propagating in free space for a total crystal thickness $L$. Figure 2(a) displays the transmission peak at $\Omega=11.954 \mathrm{GHz}$ within the photonic band gap. The quality factor of this peak is 940 . At the resonance frequency, the delay time also has a peak with $\tau_{p}=23 \mathrm{~ns}$ [Fig. 2(b)].

Next, we determined the transmission and phase information of two consecutive defects with intercavity distance $\Lambda$ $=1.34 \mathrm{~cm}$. The single mode in the previous case splitted into bonding and antibonding modes at $\omega_{\mathrm{B}}=11.833 \mathrm{GHz}$ and $\omega_{\mathrm{A}}=12.012 \mathrm{GHz}$ with $Q=1183$ and $Q=1365$, respectively [Figs. 2(c) and 2(e)]. Figures 2(d) and 2(f) show the delay time as a function of frequency for the two coupled defect case. The lifetime of these hybridized resonant modes are $\tau_{p}^{\mathrm{B}}=34 \mathrm{~ns}$ and $\tau_{p}^{\mathrm{A}}=37 \mathrm{~ns}$. In all measurements, it is observed that $\tau_{p}^{\mathrm{A}}>\tau_{p}^{\mathrm{B}}$. This observation may be explained qualitatively by exploring the properties of the corresponding modes which can be written as the superposition of the individual cavity modes at each defect side $\mathbf{E}_{\omega}^{\mathrm{B}}(\mathbf{r})=\left[\mathbf{E}_{\Omega}(\mathbf{r})\right.$

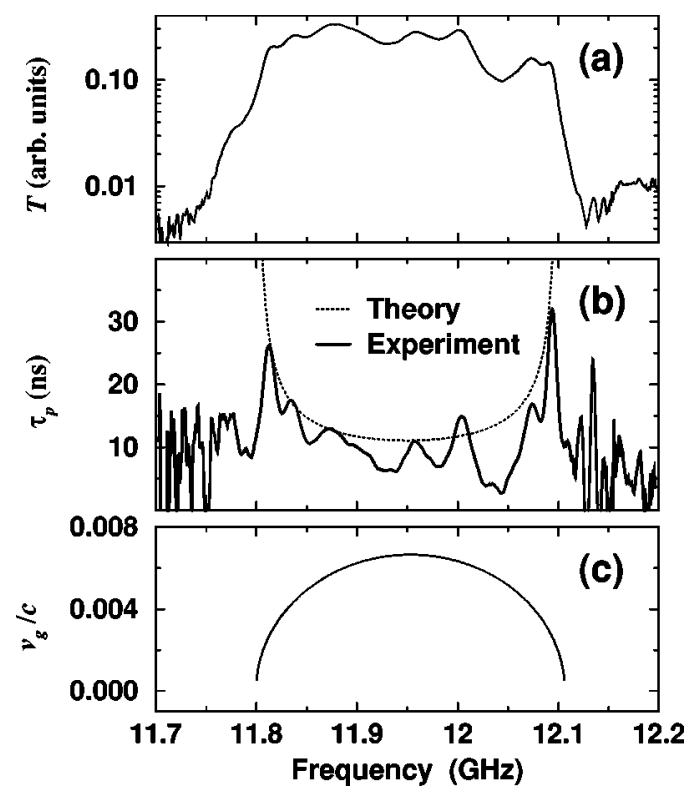

FIG. 3. (a) Transmission through ten unit cell periodic coupled defects. (b) The measured and calculated delay time for this coupled-cavity waveguide $(\mathrm{CCW})$. The photon lifetime increases rapidly at the waveguiding band edges. (c) The normalized group velocity versus frequency. Here $c$ is the speed of light in a vacuum. Photons slow down significantly at the waveguiding band edges $\left(v_{g} \rightarrow 0\right)$, and the maximum group velocity is two orders of magnitude smaller than $c$.

$\left.+\mathbf{E}_{\Omega}(\mathbf{r}-\Lambda \hat{x})\right] / \sqrt{2}$ and $\mathbf{E}_{\omega}^{\mathrm{A}}(\mathbf{r})=\left[\mathbf{E}_{\Omega}(\mathbf{r})-\mathbf{E}_{\Omega}(\mathbf{r}-\Lambda \hat{x})\right] / \sqrt{2}$. Confinement of the antibonding mode is stronger than the bonding mode, since the magnitude of the antibonding field has nonzero values only near the defect sides. (See Refs. 27 and 28 for a more quantitative analysis of bonding and antibonding modes in photonic crystals.) However, our observation still needs more theoretical investigations. It is important to note that based on our experimental results, the lifetime is directly proportional to the quality factor via $\tau_{p} \propto Q / \Omega$.

When the number of defects is increased, a guiding band which we called a coupled-cavity waveguide (CCW) band, is formed within the photonic band gap. For a ten unit cell crystal, we measured the transmission amplitude, transmission phase, and the delay time. Figure 3(a) shows the transmission-amplitude properties of the CCW band extending from $11.798 \mathrm{GHz}$ to $12.105 \mathrm{GHz}$. As shown in Fig. 3(b), the delay time has two peaks at the CCW band edges implying that the transit time for photons at the band edges through the crystal is increased significantly.

The dispersion relation $\omega_{k}$ of the periodic coupled cavities can be obtained from the transmission-phase data. By measuring the net phase difference $\varphi$ as a function of frequency $\omega$, the wave vector $k$ of the crystal can be determined directly: ${ }^{13,14}$

$$
k=\varphi / L+2 \pi \omega / c,
$$

where $L$ is the total crystal thickness, and $c$ is the speed of the light in vacuum. The dispersion relation can also be determined within the classical wave analog of the TB approximation $^{16,17,13,14}$

$$
\omega_{k}=\Omega[1+\kappa \cos (k \Lambda)],
$$


where $\Omega$ is the resonance frequency of a single defect, $\kappa$ $=-0.013$ is the TB parameter which was determined from the splitting of two coupled cavities. Combining Eqs.(1) and (2) along with definition of $\tau_{p}$, we got a formula for photon lifetime as a function of frequency:

$$
\tau_{p}(\omega)=\frac{L / \Lambda}{\Omega \sqrt{\kappa^{2}-(\omega / \Omega-1)^{2}}}+2 \pi L / c
$$

This formula shows that the lifetime of photons increases rapidly as we approach to the waveguiding band edges. Figure 3(b) displays the comparison between the experimental and the theoretical results. The measured photon lifetime is in good agreement with the calculated results. The oscillations in the Fig. 3(b) is due to the finite number of unit cell in the crystal.

Group velocity of photons along the coupled-cavity array can be deduced from the dispersion relation, i.e., Eq.(2), as $v_{g}=\nabla_{k} \omega_{k}=-\kappa \Lambda \Omega \sin (k \Lambda)$. In Fig. 3(c), the normalized group velocity is plotted throughout the CCW band. We observed that $v_{g} \rightarrow 0$ at the $\mathrm{CCW}$ band edges, and this result supports the long photon lifetime proposal.

Let us calculate the transit time of photons through the crystal of thickness $L=13.4 \mathrm{~cm}$. The average group velocity of photons is simply determined from $\bar{v}_{g}$ $=\Lambda / \pi \int_{0}^{\pi / \Lambda} v_{g}(k) d k=2|\kappa| \Lambda \Omega / \pi$. Then the transit time is given by $\tau=L / \bar{v}_{g}=\pi L / 2|\kappa| \Lambda \Omega$. It can be assumed that $\tau$ consists of the escape rate of photons from the cavities, i.e., delay time $\left(\tau_{p}\right)$ and the time spent between the cavities, i.e., tunneling time $\left(\tau_{t}\right) .^{7}$ Since $\tau_{p}$ is very long compared with $\tau_{t}$, we can neglect the tunneling time. Hence, we found that $\tau_{p} \simeq \tau \sim 10 \mathrm{~ns}$. This value agrees well with the experimental and the theoretical results in Fig. 3(b) for frequencies away from the band edges.

Finally, we investigated the delay time of a defect structure where the whole rod is removed from each unit cell of the crystal. ${ }^{13,14}$ We measured the delay time corresponding to a ten unit cell coupled-cavity waveguide. The localization

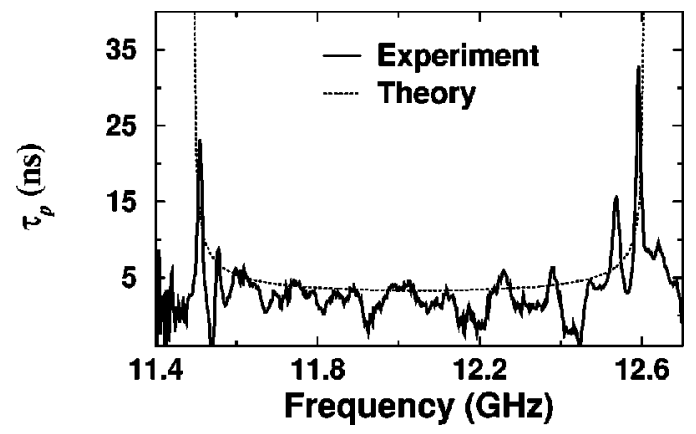

FIG. 4. The measured and calculated delay time for the single rod removed CCW. The photon lifetime also increases drastically at the waveguiding band edges.

volume is bigger than in the previous case, and therefore the photon lifetime for a single cavity is around $5 \mathrm{~ns}$. As shown in Fig. 4, the delay time increases drastically at the CCW band edges and agrees well with the calculated result by using Eq. (3). In this case, the group velocity also tends towards zero at the edges [see Fig. (4) of Ref. 13].

Physically, the heavy photons in the photonic band gap structures are analogous to the electrons in semiconductors having energies near the band edges. The corresponding eigenfunctions are standing waves rather than the running waves, and therefore the effective mass of electrons becomes very large. ${ }^{29}$

In summary, we have presented our experimental observation of heavy photons at the coupled-cavity waveguiding band edges in the 3D photonic crystals. The theoretical analysis based on the classical wave analog of the tightbinding scheme is in good agreement with experimental results. Extremely small group velocity offers a promising tool to increase the spontaneous emission rate, efficiency of nonlinear processes, and for optoelectronic devices.

This work was supported by Turkish Department of Defense Grant No. KOBRA-001, NATO Grant No. SfP971970, and National Science Foundation Grant No. INT-9820646. The authors thank B. Temelkuran, A. S. Shumovsky, C. Yalabik, and S. Ciraci for many helpful discussions.
*Author to whom correspondence should be addressed. Electronic address: bayindir@fen.bilkent.edu.tr

${ }^{1}$ For a recent review, see articles in Photonic Band Gap Materials, edited by C. M. Soukoulis (Kluwer, Dordrecht, 1996).

${ }^{2}$ J. D. Joannopoulos, R. D. Meade, and J. N. Winn, Photonic Crystals: Molding the Flow of Light (Princeton University Press, Princeton, NJ, 1995).

${ }^{3}$ S. John, Phys. Rev. Lett. 58, 2486 (1987).

${ }^{4}$ Z. Q. Zhang et al., Phys. Rev. Lett. 81, 5540 (1998).

${ }^{5}$ A. M. Steinberg, P. G. Kwiat, and R. Y. Chiao, Phys. Rev. Lett. 71, 708 (1993); A. M. Steinberg and R. Y. Chiao, Phys. Rev. A 51, 3525 (1995).

${ }^{6}$ Ch. Spielmann, R. Szipocs, A. Stingl, and F. Krausz, Phys. Rev. Lett. 73, 2308 (1994).

${ }^{7}$ G. Nimtz, in Tunneling and Its Implications, edited by A. Ranfagin (World Scientific, Singapore, 1997).

${ }^{8}$ P. Pereyra, Phys. Rev. Lett. 84, 1772 (2000).

${ }^{9}$ E. Yablonovitch, Phys. Rev. Lett. 58, 2059 (1987).

${ }^{10}$ M. D. Tocci et al., Phys. Rev. A 53, 2799 (1996).
${ }^{11}$ J. M. Gerard et al., Phys. Rev. Lett. 81, 1110 (1998).

${ }^{12}$ E. Yablonovitch et al., Phys. Rev. Lett. 67, 3380 (1991).

${ }^{13}$ Mehmet Bayindir, B. Temelkuran, and E. Ozbay, Phys. Rev. Lett. 84, 2140 (2000).

${ }^{14}$ Mehmet Bayindir, B. Temelkuran, and E. Ozbay, Phys. Rev. B 61, R11 855 (2000).

${ }^{15}$ E. Lidorikis, M. M. Sigalas, E. N. Economou, and C. M. Soukoulis, Phys. Rev. Lett. 81, 1405 (1998).

${ }^{16}$ N. Stefanou and A. Modinos, Phys. Rev. B 57, 12127 (1998).

${ }^{17}$ A. Yariv, Y. Xu, R. K. Lee, and A. Scherer, Opt. Lett. 24, 711 (1999).

${ }^{18}$ J. P. Albert, C. Jouanin, D. Cassagne, and D. Bertho, Phys. Rev. B 61, 4381 (2000).

${ }^{19}$ J. P. Dowling, M. Scalora, M. J. Bloemer, and C. M. Bowden, J. Appl. Phys. 75, 1896 (1994).

${ }^{20}$ K. Sakoda, Opt. Express 4, 167 (1999), and references therein.

${ }^{21}$ J. P. Dowling and C. M. Bowden, J. Mod. Opt. 41, 345 (1994).

${ }^{22}$ Yu. A. Vlasov, S. Petit, G. Klein, B. Honerlage, and Ch. Hirlimann, Phys. Rev. E 60, 1030 (1999). 
${ }^{23}$ K. M. Ho, C. T. Chan, C. M. Soukoulis, R. Biswas, and M. M. Sigalas, Solid State Commun. 89, 413 (1994)

${ }^{24}$ E. Ozbay, J. Opt. Soc. Am. B 13, 1945 (1996).

${ }^{25}$ E. Ozbay and B. Temelkuran, Appl. Phys. Lett. 69, 743 (1996).

${ }^{26}$ E. Ozbay, G. Tuttle, M. M. Sigalas, C. M. Soukoulis, and K. M. Ho, Phys. Rev. B 51, 13961 (1995).
${ }^{27}$ M. I. Antonoyiannakis and J. B. Pendry, Europhys. Lett. 40, 613 (1997).

${ }^{28}$ M. I. Antonoyiannakis and J. B. Pendry, Phys. Rev. B 60, 2363 (1999).

${ }^{29}$ C. Kittel, Introduction to Solid State Physics, 7th ed. (John Wiley \& Sons, New York, 1996), p. 209. 